\title{
ON $f$-RINGS WITH THE ASCENDING CHAIN CONDITION ${ }^{1}$
}

\author{
F. W. ANDERSON
}

Introduction. In [1] Birkhoff and Pierce obtain the structure of $f$-rings ${ }^{2}$ which have no nonzero nilpotent elements and satisfy the descending chain condition for $l$-ideals. More recently, D. G. Johnson [4] gives the structure of $J$-semi-simple $f$-rings ( $\$ 2)$ with the descending chain condition for $l$-ideals. In this note our principal aim is to give the structure of $f$-rings with various ascending chain conditions. We first show (Theorem 1) that in $f$-rings the ascending and descending chain conditions for closed $l$-ideals are equivalent and that an $f$-ring with these conditions can be characterized as a subdirect sum of finitely many totally ordered rings. Next (Theorem 2) we specialize to the case of $f$-rings with no nonzero nilpotent elements. In $\$ 2$ we consider $J$-semi-simple $f$-rings. For these $f$-rings we show (Theorem 4 ) that the ascending and descending chain conditions for $l$-ideals and for closed $l$-ideals are all equivalent.

In [3] Goldie proves that a semi-simple ring with the ascending chain condition for ideals is a subdirect sum of a finite number of semi-simple prime rings. An examination of the proof of this result shows that he proves even more, namely, that a semi-prime ring with the ascending chain condition for annihilator ideals is a subdirect sum of a finite number of prime rings. The results of this note provide $f$-ring analogues of the results of [3], and the techniques we employ are patterned after those of Goldie.

1. Chain conditions for closed $l$-ideals. Let $A$ be an $f$-ring. By an l-ideal of $A$ we mean a ring ideal $I$ such that for all $a, b \in A$ if $b \in I$ and $|a| \leqq|b|$, then $a \in I$. If $S$ is a nonempty subset of $A$, then we set

$$
S^{\perp}=\{a \in A ;|a| \wedge|x|=0(x \in S)\} .
$$

It is clear that: (i) $S^{\perp}$ is an $l$-ideal of $A$; (ii) $S \cap S^{\perp}=\{0\}$; (iii) $S \subseteq S^{\perp \perp}$; and (iv) $S^{\perp}$ is contained in both the left and right (ring) annihilators of $S$. We say that $S$ is complemented in case $S^{\perp} \neq\{0\}$ and closed in case $S=S^{\perp \perp}$.

Presented to the Society, June 16,1961; received by the editors May 15, 1961 and, in revised form, August 3,1961.

1 This work was supported by a grant from the National Science Foundation.

2 An f-ring is a lattice-ordered ring in which $a \wedge b=0$ and $c \geqq 0$ imply $c a \wedge b=$ $a c \wedge b=0$. In [1] Birkhoff and Pierce, who introduced the concept, prove that $f$-rings are characterizable as subdirect sums of totally ordered rings. For the general theory of lattice-ordered rings and of $f$-rings see Birkhoff and Pierce [1], Johnson [4], and Pierce $[5]$. 
LEмма 1. If $I$ is a nonzero $l$-ideal of an f-ring $A$, then the following statements are equivalent:

(1) $I$ is totally ordered (as a sub f-ring of $A$ );

(2) $I^{\perp}$ is a maximal closed l-ideal;

(3) $A / I^{\perp}$ is totally ordered.

Proof. (1) $\Rightarrow(3)$. Assume that $I$ is totally ordered. Then if $a, b \notin I^{\perp}$ are positive, there exists an element $c \in I$ such that $a \wedge c>0$ and $b \wedge c$ $>0$. Since $I$ is totally ordered and an $l$-ideal of $A,(a \wedge b) \wedge c>0$. Thus $a \wedge b \notin I^{\perp}$, and so, $A / I^{\perp}$ is totally ordered.

$(3) \Rightarrow(2)$. Assume $A / I^{\perp}$ is totally ordered. To see that $I^{\perp}$ is a maximal closed $l$-ideal, it will suffice to show that if $a \notin I^{\perp}$, then $\{a\}^{\perp} \cap I^{\perp \perp}$ $=\{0\}$ since the closed $l$-ideal generated by $\{a\} \cup I^{\perp}$ is

$$
\left(\{a\} \cup I^{\perp}\right)^{\perp \perp}=\left(\{a\}^{\perp} \cap I^{\perp \perp}\right)^{\perp} .
$$

But if $x \in\{a\}^{\perp} \cap I^{\perp \perp}$, then since $A / I^{\perp}$ is totally ordered, since $|x| \wedge|a|=0$, and since $a \notin I^{\perp}$, we have $x \in I^{\perp}$. Therefore $\{a\}^{\perp} \cap I^{\perp \perp}$ $\subseteq I^{\perp} \cap I^{\perp \perp}=\{0\}$.

$(2) \Rightarrow(1)$. If $I$ is not totally ordered, then $I^{\perp \perp}$ is not totally ordered. So there exist nonzero elements $a, b \in I^{\perp \perp}$ such that $a \wedge b=0$. If $J$ is the $l$-ideal generated by $I^{\perp} \cup\{a\}$, then $b \in J^{\perp}$. Thus $J^{\perp \perp}$ is a proper closed $l$-ideal properly containing $I^{\perp}$; hence, $I^{\perp}$ is not a maximal closed $l$-ideal.

LEMMA 2. If $M$ and $N$ are maximal closed $l$-ideals of an $f$-ring $A$, then $M \neq N$ if and only if $N^{\perp} \subseteq M$.

Proof. By Lemma $1, N^{\perp}$ is totally ordered. Since $M$ is closed, it is clear then that either $N^{\perp} \subseteq M$ or $N^{\perp} \subseteq M^{\perp}$. So if $N^{\perp} \Phi M$, then $M \subseteq N$ and, by the maximality of $M, M=N$. Conversely, if $M=N$, then $N^{\perp}=M^{\perp} \Phi M$ since $M^{\perp} \neq\{0\}$.

In general, an $f$-ring need not have any maximal closed $l$-ideals. An example of such an $f$-ring is the $f$-ring of all continuous real-valued functions on $[0,1]$. Also, a maximal closed $l$-ideal need not be a maximal $l$-ideal. For example, let $Q[\lambda]$ be the ring of polynomials in one indeterminate over the rational field ordered lexicographically $\left(1>\lambda>\lambda^{2}>\cdots\right) \cdot{ }^{3}$ Then $\{0\}$ is a maximal closed $l$-ideal but not a maximal $l$-ideal.

LEMMA 3. If $A$ is an f-ring satisfying the ascending chain condition for closed l-ideals, then every complemented l-ideal of $A$ is contained in a maximal closed l-ideal.

3 See Johnson [4, p. 172]. 
Proof. If $I$ is a complemented $l$-ideal, then $I^{\perp \perp}$ is a proper closed $l$-ideal containing $I$.

LEMMA 4. If $A$ is an f-ring satisfying the ascending chain condition for closed l-ideals, then the set Th of maximal closed l-ideals of $A$ is finite and $\cap \pi \mathrm{N}=\{0\}$.

Proof. By Lemma 3, $\mathfrak{T} \neq \varnothing$. We show first that $\cap \mathfrak{T}=\{0\}$. For if $\cap \Re \neq\{0\}$, then $(\cap \Re)^{\perp}$ is complemented. Thus, by Lemma 3, there is an $M \in \mathscr{M}$ with $(\cap \mathscr{T})^{\perp} \subseteq M$. Since this implies $M^{\perp} \subseteq M$, we have the contradiction $M^{\perp}=\{0\}$; hence $\cap \mathscr{T}=\{0\}$.

Now using the ascending chain condition for closed $l$-ideals, we see that there exist $M_{1}, \cdots, M_{n} \in \mathscr{N}$ such that

$$
M^{\perp} \subseteq\left(M_{1} \cap \cdots \cap M_{n}\right)^{\perp} \quad(M \in \mathscr{M}) .
$$

Thus, $M_{1} \cap \cdots \cap M_{n}=\{0\}$. If $M \in \mathscr{T}$ and $M \neq M_{i}(i=1, \cdots, n)$, then, by Lemma $2, M^{\perp} \subseteq M_{1} \cap \cdots \cap M_{n}$ contrary to $M^{\perp} \neq\{0\}$. Therefore, $\mathfrak{T}=\left\{M_{1}, \cdots, M_{n}\right\}$.

ThEOREM 1. For an f-ring $A$ the following statements are equivalent:

(1) $A$ has the ascending chain condition for closed l-ideals.

(2) $A$ has the descending chain condition for closed l-ideals.

(3) $A$ is isomorphic to a subdirect sum of a finite number of totally ordered rings.

Proof. The equivalence of (1) and (2) is clear since the mapping $I \rightarrow I^{\perp}$ is a dual automorphism of the lattice of closed $l$-ideals of $A$. The implication $(3) \Rightarrow(1)$ is trivial. Finally, the implication $(1) \Rightarrow(3)$ follows immediately from Lemmas 1 and 4.

If $A$ is an $f$-ring, then the set $N(A)$ of all nilpotent elements of $A$ is an $l$-ideal called the l-radical of $A$ [1]. Clearly, $N(A / N(A))=\{0\}$ and $N(I)=N(A) \cap I$ for any $l$-ideal $I$ of $A$. The $l$-radical $N(A)$ of $A$ can also be characterized [5] as the intersection of all prime $l$-ideals of $A$. Recall [4] that in an $f$-ring $A$ an $l$-ideal $P$ is prime if and only if for all $a, b \in A, a b \in P$ implies $a \in P$ or $b \in P$. This is also equivalent to the property: $A / P$ is totally ordered with no nonzero divisors of zero.

If $A$ is an $f$-ring with $N(A)=\{0\}$, then it follows that if $S \subseteq A$ is nonvoid, its left annihilator, right annihilator, and $S^{\perp}$ coincide [1, p. 63].

Lemma 5. Let $A$ be an f-ring with $N(A)=\{0\}$ and let $P$ be an l-ideal of $A$. Then $P$ is a complemented prime $l$-ideal if and only if $P$ is a maximal closed l-ideal. 
Proof. If $P$ is a complemented prime $l$-ideal, then $P^{\perp} \neq 0$, whence $P^{\perp} \Phi P$. Since $P^{\perp} P^{\perp \perp}=\{0\}$, we have that $P^{\perp \perp} \subseteq P$, so that $P$ is closed. Also, since $P$ is prime, $A / P$ is totally ordered. Therefore, by Lemma 1 (with $I=P^{\perp}$ ), we have that $P=P^{\perp \perp}$ is a maximal closed $l$-ideal.

Conversely, it will suffice to show that if $P$ is maximal closed, then it is prime. But in this case $P^{\perp}$ is totally ordered by Lemma 1 , so that if $a, b \notin P$, there exists a $c \in P^{\perp}$ such that $|a| \wedge|c| \neq 0$ and $|b| \wedge|c|$ $\neq 0$. Now $N\left(P^{\perp}\right)=N(A) \cap P^{\perp}=\{0\}$, so that $P^{\perp}$ is a prime $f$-ring. Therefore, since $|a| \wedge|c|,|b| \wedge|c| \in P^{\perp}$, we have

$$
0 \neq(|a| \wedge|c|)(|b| \wedge|c|) \leqq|a||b|=|a b| .
$$

Since $P^{\perp} \cap P=\{0\}$, it follows that $(|a| \wedge|c|)(|b| \wedge|c|) \notin P$. Thus $a b \notin P$, and $P$ is prime.

Now from Lemma 4, Lemma 5 , and Theorem 1 we readily conclude

THEOREM 2. Let $A$ be an f-ring with $N(A)=\{0\}$. Then the following statements are equivalent:

(1) A has the ascending chain condition for closed l-ideals.

(2) $A$ has the descending chain condition for closed l-ideals.

(3) $A$ is isomorphic to a subdirect sum of a finite number of totally ordered rings having no nonzero divisors of zero.

As we have now seen the ascending and descending chain conditions for closed $l$-ideals are equivalent in any $f$-ring. However, even for $f$-rings with zero $l$-radical the ascending and descending chain conditions for $l$-ideals need not be equivalent. For example, the $f$-ring $Q[\lambda]$, which has zero $l$-radical, satisfies the ascending but not the descending chain condition for $l$-ideals. Note, however, that if $N(A)=\{0\}$ and if $A$ satisfies the descending chain condition for $l$-ideals, then $A$ is isomorphic to a finite direct sum of $l$-simple totally ordered rings [1, Theorem 17] and therefore satisfies the ascending chain condition for $l$-ideals.

We also observe that in Theorems 1 and 2 the "subdirect sum" of statement (3) cannot be strengthened to "direct sum." For let $A$ be the sub $f$-ring of the direct sum of two copies of $Q[\lambda]$ defined by

$$
A=\{(f, g) ; f, g \in Q[\lambda] \text { with } f(0)=g(0)\}
$$

Then $N(A)=\{0\}$ and $A$ has the ascending chain condition for closed $l$-ideals, but $A$ cannot be isomorphic to a direct sum of totally ordered rings.

2. Chain conditions in $J$-semi-simple $f$-rings. An $l$-ideal $P$ of an $f$-ring $A$ is $l$-primitive if and only if $A / P$ is an $l$-simple ordered ring 
with identity. Thus an $l$-primitive $l$-ideal is prime. The $J$-radical, $J(A)$, of $A$ is the intersection of all $l$-primitive $l$-ideals of $A$. Clearly $N(A) \subseteq J(A)$. If $J(A)=\{0\}$, then $A$ is $J$-semi-simple. ${ }^{4}$

The example $Q[\lambda]$ shows that in $f$-rings with zero $l$-radical closed prime $l$-ideals need not be $l$-primitive. However, for $J$-semi-simple $f$ rings we have

Lemma 6. If $P$ is a closed prime l-ideal of a J-semi-simple f-ring $A$, then $P$ is an l-primitive l-ideal and

$$
A=P \oplus P^{\perp} \text {. }
$$

Proof. By Lemmas 1 and $5, P^{\perp}$ is totally ordered and so, since $J\left(P^{\perp}\right)=J(A) \cap P^{\perp}=\{0\}\left[4\right.$, p. 188], $P^{\perp}$ is an $l$-primitive $f$-ring. Let $e \in P^{\perp}$ be the identity for $P^{\perp}$. Then, since $P$ is the right ring annihilator of $P^{\perp}$,

$$
a=(a-e a)+e a \in P+P^{\perp}
$$

for all $a \in A$. Thus, $A=P+P^{\perp}$ and since $P \cap P^{\perp}=\{0\}$, this sum is direct. Therefore $A / P$ is isomorphic to $P^{\perp}$ and $P$ is an $l$-primitive $l$-ideal.

THEOREM 3. Let $A$ be a J-semi-simple f-ring satisfying the ascending chain condition for closed l-ideals. Then the set $P$ of closed prime l-ideals of $A$ coincides with the set of l-primitive l-ideals of $A$, and $A$ is the direct sum of the l-ideals $P^{\perp}(P \in \mathcal{P})$.

Proof. By Lemmas 4 and $5, \odot$ is finite. Let $\odot=\left\{P_{1}, \cdots, P_{n}\right\}$. By Lemmas 2 and 6 the sum

$$
P_{1}^{\perp}+\cdots+P_{n}^{\perp}
$$

is direct and each $P_{t}^{\perp}$ is an $l$-primitive $f$-ring. If $e_{i} \in P_{i}^{\perp}$ is the identity of $P_{i}^{\perp}$, then for each $a \in A$

$$
a-\sum_{i=1}^{n} e_{i} a \in P_{1} \cap \cdots \cap P_{n}
$$

By Lemma 4 this implies that

whence

$$
a=\sum_{i=1}^{n} e_{i} a
$$

$$
A=P_{1}^{\perp} \oplus \cdots \oplus P_{n}^{\perp}
$$

4 The notions of $l$-primitivity and of the $J$-radical as well as the structure theory of $J$-semi-simple $f$-rings are due to Johnson [4]. 
To complete the proof it will suffice, in view of Lemma 6, to show that each $l$-primitive $l$-ideal $P$ of $A$ is one of the $P_{i}(i=1, \cdots, n)$. But since $P$ is proper $P_{i}^{\perp} \Phi P$ for some $i$ and so, since $P$ is prime $P_{i} \subseteq P$. However, $P_{i}$ is $l$-primitive, hence maximal $\left[4\right.$, p. 187]; thus $P_{i}=P$.

Theorem 4. For a $J$-semisimple f-ring $A$ the following statements are equivalent:

(1) $A$ has the ascending chain condition for closed l-ideals.

(2) $A$ has the descending chain condition for closed l-ideals.

(3) $A$ has the ascending chain condition for l-ideals.

(4) $A$ has the descending chain condition for l-ideals.

(5) $A$ is isomorphic to the direct sum of a finite set of l-simple totally ordered rings with identity.

Proof. The implication $(1) \Rightarrow(5)$ is by Theorem 3. Also (1) $\Leftrightarrow(2)$ by Theorem 1. By Theorem II.5.8 of [4] we have $(4) \Leftrightarrow(5)$. Since $(5) \Rightarrow(3) \Rightarrow(1)$ are trivial, the proof is complete.

3. Remarks. If an $f$-ring $A$ satisfies the ascending chain condition for $l$-ideals (closed $l$-ideals), then each $l$-ideal (closed $l$-ideal) of $A$ is principal. For the $l$-ideal (closed $l$-ideal) generated by $\left\{a_{1}, \cdots, a_{n}\right\}$ is also generated by $\left|a_{1}\right| \vee \cdots \vee\left|a_{n}\right|$. Conversely, if each $l$-ideal of an $f$-ring $A$ is principal, then $A$ satisfies the ascending chain condition for $l$-ideals. Such a converse is not valid, however, for closed $l$-ideals. For example, in the $f$-ring of all real-valued functions on the integers every closed $l$-ideal is principal, but this $f$-ring clearly does not have the ascending chain condition for closed $l$-ideals.

If $A$ is an arbitrary ring with the descending chain condition for right ideals, then $A$ has the ascending chain condition for right ideals if and only if the additive group of $A$ contains no $p^{\infty}$ group (Fuchs [2]). Certainly the additive group of an $f$-ring has this property since this group must be torsion free. However, let $A$ be the ring whose additive group is that of $Q[\lambda]$ and with multiplication defined by

$$
\left(\sum_{i=0}^{m} a_{i} \lambda^{i}\right)\left(\sum_{j=0}^{n} b_{j} \lambda^{j}\right)=\sum_{i=0}^{m}\left(b_{0} a_{i}\right) \lambda^{i}+\sum_{j=1}^{n}\left(a_{0} b_{j}\right) \lambda^{j}
$$

Order $A$ by

$$
a_{0}+a_{1} \lambda+\cdots+a_{m} \lambda^{m}>0
$$

in case $a_{0}>0$ or $a_{0}=0$ and $a_{m}>0$. Then $A$ is a commutative $f$-ring with identity which satisfies the descending but not the ascending chain condition for $l$-ideals.

Finally, it is known $[4$, p. 213$]$ that in an $f$-ring with zero $l$-radical 
the descending chain condition on $l$-ideals and the descending chain condition on right $l$-ideals are equivalent. The corresponding statement for ascending chain conditions fails. For it can be shown that in an example due to Johnson [4, pp. 208-209] we have an $f$-ring with zero $l$-radical which satisfies the ascending chain condition for $l$-ideals but not the ascending chain condition for right $l$-ideals. ${ }^{6}$

\section{REFERENCES}

1. G. Birkhoff and R. S. Pierce, Lattice-ordered rings, An. Acad. Brasil. Ci. 28 (1956), 41-69.

2. L. Fuchs, Wann folgt die Maximalbedingung aus der Minimalbedingung?, Arch. Math 8(1957), 317-319.

3. A. W. Goldie, Decompositions of semi-simple rings, J. London Math. Soc. 31 (1956), 4048.

4. D. G. Johnson, $A$ structure theory for a class of lattice-ordered rings, Acta Math. 104 (1960), 163-215.

5. R. S. Pierce, Radicals in function rings, Duke Math. J. 23 (1956), 253-261.

UNIVERSITY OF OREgon

- In the $f$-ring of this example the principal right $l$-ideals generated by the elements $x a, x^{2} a, \cdots$ form a properly ascending chain. 